\title{
Design of Calcium Phosphate Scaffolds with controlled Simvastatin release by Plasma polymerisation
}

Cristina Canal $^{1,2 \ddagger}$, , Kanupriya Khurana ${ }^{1,2 \ddagger}$, Sara Gallinetti ${ }^{1,2}$, Sudhir Bhatt ${ }^{3,4}$, Jérome Pulpytel $^{3,4}$, Farzaneh Arefi-Khonsari ${ }^{3,4}$, Maria-Pau Ginebra ${ }^{1,2}$

${ }^{1}$ Biomaterials, Biomechanics and Tissue Engineering Group, Dpt. Materials Science and Metallurgy, Technical University of Catalonia (UPC), Av. Diagonal 647, 08028 Barcelona, Spain

${ }^{2}$ Research Centre in NanoEngineering (CrNE), UPC

${ }^{3}$ Sorbonne Universités, UPMC Univ Paris 06, UMR8235, LISE, 4 place Jussieu, 75252 Paris, France

${ }^{4}$ CNRS, UMR8235, LISE, 4 place Jussieu, 75252 Paris, France

*corresponding author: cristina.canal@upc.edu

\$ Both authors contributed equally

KEYWORDS: Calcium Phosphate, scaffolds, PCL-PEG plasma polymerisation, cements, ceramics, controlled drug release 


\begin{abstract}
Calcium Phosphates (CaPs) have excellent bone regeneration capacity, and their combination with specific drugs is of interest because it allows adding new functionalities. In CaPs, drug release is mainly driven by diffusion, which is strongly affected by the porosity of the matrix and the drug-material interaction. Therefore, it is very difficult to tune their drug release properties beyond their intrinsic properties. Furthermore, when the CaPs are designed as scaffolds, the increased complexity of the macrostructure further complicates the issue.
\end{abstract}

This work investigates for the first time the use of biocompatible plasma-polymers to provide a tool to control drug release from drug-loaded $\mathrm{CaP}$ scaffolds with complex surfaces and intricate 3D structure. Two different CaPs were selected displaying great differences in microstructure: low-temperature CaPs (Calcium-deficient hydroxyapatite cements, CDHA) and sintered $\mathrm{CaP}$ ceramics ( $\beta$-Tricalcium Phosphate, $\beta$-TCP). The deposition of PCL-co-PEG (1:4) copolymers on CaPs was achieved by a low pressure plasma process, which allowed coating the inner regions of the scaffolds up to a certain depth. The coating covered the micro and nanopores of the CaPs surface and produced complex geometries presenting a nano and micro rough morphology which lead to low wettability despite the hydrophilicity of the copolymer. Plasma coating with PCL-coPEG on scaffolds loaded with Simvastatin acid (potentially osteogenic and angiogenic) allowed delaying and modulating the drug release from the bone scaffolds depending on the thickness of the layer deposited, which, in turn depends on the initial specific surface area of the CaP. 


\section{Introduction}

Calcium Phosphates $(\mathrm{CaP})$ are excellent candidates in bone replacement due to their similarity to the mineral phase of bone and potential resorbability $[1,2]$. Their combination with different types of drugs allows providing them with additional functionalities, in addition to their excellent osteoconductivity and osteogenicity. However, in CaPs a part of the loaded drug often remains trapped in the matrix [3, 4]. This could be partly solved by introduction of macroporosity to the material, by production of different kinds of scaffolds $[5,6]$. In any case - both in bulk materials as in scaffolds - the performance of these ceramic matrices as drug delivery systems is tightly linked to their inherent porosity and pore size distribution features, which is dependent on the fabrication method followed [3]. Thus, whereas biomimetic calcium phosphate cements exhibit a high porosity, ranging from the nanometric to the micrometric scale, the porosity in sintered $\mathrm{CaP}$ ceramics tends to be micrometric in size.

Thus, a common problem is that drug release cannot be tuned beyond their intrinsic capacity, which is related with their porosity. Therein, adapting the release profile to specific pathologies requiring a certain rate of delivery poses a problem. Coating of the material's surface could be a solution. However, once the ceramic matrices are loaded with drugs, coating by conventional wet methods can lead to loss of drug from the material to the coating media.

In those cases, a promising novel approach to tune the drug release kinetics can be found in low temperature plasma technologies. Low temperature plasma, herein plasma, can be defined as a particular state of a gas or mixture of gases containing a mixture of ions, free radicals, electrons, excited molecules, UV and visible radiation that preserves electrical neutrality. This reactive medium can modify the first 
nanometers of the surface of the material without altering its bulk properties. Roughly, the three main effects of plasmas on the surface of a material are: i) functionalization or grafting (covalent bonding of new chemical species); ii) etching (removal of surface material); and iii) thin film deposition (deposition of thin layers).

These effects may be employed to tailor drug release; Recent works $[7,8]$ have shown that surface functionalization of polyamide 6.6 fibres can improve the amount of anti-inflammatories and lipolithic agents released due to improved interaction of the materials with the surrounding media.

Another approach which can be used to slow down the drug release profile is by creation of thin films by plasma polymerisation on the surface of the materials, acting as barrier for its release. Coating by plasma polymerization refers to the deposition of polymer films due to the excitation of an organic monomer in the gas state and subsequent deposition and polymerization of the excited species on the surface of a substrate [9].

Few works have investigated plasma polymerization to produce an overlayer to control the release kinetics of drugs placed on the surface of solid carrier surfaces. Vasilev et al. have coated Vancomycin contained in nanoporous anodic aluminium oxide with allyllamine plasma layers [10]. In another work, quartz surfaces were employed as supports for a first n-heptylamine plasma polymer layer, to allow suitable wettability of Levofloxacin which was subsequently coated with a second n-heptylamine layer [11]. Similarly, quartz surfaces were employed as supports for model dye molecules (Methylene Blue) or anticancer agents (Cisplatin) and were plasma coated with multilayered biodegradable Poly caprolactone-co- 
polyethyleneglycol (PCL-co-PEG) coatings [12]. Both authors have shown that by gradually increasing the barrier layer thickness with plasma polymerization deposition time, the amount of drug released diminished. In all cases, simple model surfaces have been employed.

However, coating of complex surfaces in 3D scaffold structures and being able to fine tune drug release from them is far from being obvious. Therefore, it is the main aim of this work to show the potential of plasma polymerization in drug modulation from complex ceramic scaffolds for bone regenerative applications. These scaffolds were obtained by foaming of a calcium phosphate cement, and contained different levels of micro and nanoporosity, depending on the subsequent treatment applied, namely low-temperature setting to yield Calcium Deficient Hydroxyapatite (CDHA) or high-temperature sintering to produce $\beta$-Tricalcium Phosphate ( $\beta$-TCP). Given the great differences on the surface texture of both ceramic materials, in the first stage, we investigated the deposition of biocompatible PCL-co-PEG polymer layers on 2D discs. Subsequently, the level of complexity was increased introducing 3D scaffolds, and the coating of the surface and the penetration of the plasma-polymers within the scaffolds was ascertained. To evaluate the possibilities of these layers on drug release, Simvastatin acid (SVA), was incorporated to the CaP scaffolds. In local drug delivery to the bone site from CaPs, different drugs are of interest [3]. SVA has shown potential action on bone and blood vessel formation [13-14], which is of clear interest in views of improving bone healing. Thus, to tailor the SVA release from the scaffolds plasma polymerisation of a biocompatible and biodegradable copolymer of PCL-co-PEG was evaluated as barrier layer in different conditions, and its potential effect on modulating the drug release kinetics was assessed. 


\section{Experimental}

\subsection{Materials}

$\varepsilon$-Caprolactone ( $\varepsilon-\mathrm{CL}$, Purity: 97\%, MW: 114 , Empirical formula: $\mathrm{C}_{6} \mathrm{H}_{10} \mathrm{O}_{2}$ ), and the cyclic esther monomer Diethylene glycol dimethyl ether (DEGME, Purity: 99.5\%, MW: 134.17, linear formula: $\left.\left(\mathrm{CH}_{3} \mathrm{OCH}_{2} \mathrm{CH}_{2}\right)_{2} \mathrm{O}\right)$ were purchased from Sigma Aldrich, France and used in this study without further purifications. Simvastatin $(\geq 97 \%$, MW:418.57, Sigma-Aldrich) was used as the precursor of Simvastatin acid (SVA), which was prepared according to [15].

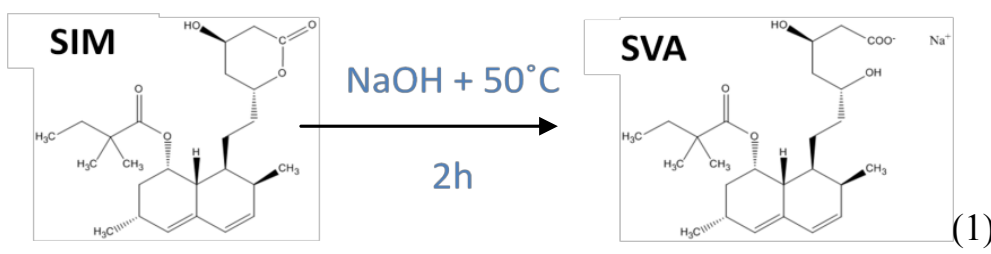

$\alpha$-TCP was used as starting material for the preparation of the powder phase of the cement, and was obtained by heating in a furnace (Hobersal CNR-58) in air, an appropriate mixture of calcium hydrogen phosphate $\left(\mathrm{CaHPO}_{4}\right.$, Sigma Aldrich) and calcium carbonate $\left(\mathrm{CaCO}_{3}\right.$, Sigma-Aldrich) at $1400^{\circ} \mathrm{C}$ for $15 \mathrm{~h}$ followed by quenching in air. $\alpha$-TCP powder was milled in an agate ball mill (Pulverisette 6, Fritsch GmbH) with 10 balls $(\mathrm{d}=30 \mathrm{~mm})$ for $15 \mathrm{~min}$ at $450 \mathrm{rpm}$ and blended with $2 \% \mathrm{wt}$ of precipitated hydroxyapatite (PHA)(HA; BP-E341, Merck, Germany), which was added as a seed. The liquid phase employed in the preparation of cements consisted of a $2.5 \%$ solution of $\mathrm{Na}_{2} \mathrm{HPO}_{4}$ (Panreac) in MilliQ water, while the liquid phase used to prepare scaffolds was a solution of $1 \mathrm{wt} \%$ of Polysorbate 80 , herein Tween 80 (Polysorbate 80, Sigma Aldrich, USA) in distilled water. 10 wt $\%$ Pluronic F-127 (Sigma Aldrich, USA) was blended with the solid phase in the preparation of the scaffolds. 


\subsection{Preparation of low temperature and sintered CaP ceramic discs and scaffolds}

$\mathrm{CaP}$ discs were used for the characterization of the polymer layer obtained. In the first place, $\mathrm{CaP}$ cements were prepared with a liquid-to-powder $(\mathrm{L} / \mathrm{P})$ ratio of $0.35 \mathrm{~mL} / \mathrm{g}$. The powder phase was mixed with the liquid phase in a mortar for about $1 \mathrm{~min}$ and then transferred into disc moulds of 2 x $15 \mathrm{~mm}$. Samples were allowed to set in Ringer's solution $(0.9 \% \mathrm{NaCl})$ for 7 days to obtain Calcium-Deficient Hydroxyapatite (CDHA) discs.

To obtain $\beta$-Tricalcium Phosphate ( $\beta$-TCP) ceramic discs, the CDHA samples were sintered in an oven (Hobersal), in air, by heating for $2.5 \mathrm{~h}$ up to $400^{\circ} \mathrm{C}$, and then for $2.20 \mathrm{~h}$ up to $110^{\circ} \mathrm{C}$ where samples were maintained for $9 \mathrm{~h}$. Cooling was achieved naturally.

Calcium phosphate scaffolds were prepared by foaming, using a L/P ratio of $0.55 \mathrm{~mL} / \mathrm{g}$. The powder phase was a mixture of $\alpha$-TCP containing $2 \mathrm{wt} \%$ of PHA and $10 \mathrm{wt} \%$ Pluronic F-127. The liquid phase was a solution of $1 \%$ wt of Tween 80 in water. The foams were injected into moulds ( $6 \mathrm{~mm}$ diameter $\mathrm{x} 12 \mathrm{~mm}$ height) and allowed to set as described. To obtain $\beta$-TCP scaffolds, the CDHA scaffolds were sintered applying the same protocol used for the $\beta$-TCP ceramic discs.

\subsection{Methods}

\subsubsection{Plasma polymerization of PCL-co-PEG coatings}

PCL-co-PEG coatings were fabricated in a low pressure inductively excited radio frequency-tubular quartz plasma reactor system $(5 \mathrm{~cm}$ diameter, $40 \mathrm{~cm}$ length, base pressure of $3 \times 10^{-2}$ mbar). The schematics of plasma deposition setup and technical 
details of the process have been provided in earlier works $[12,16,17]$. Briefly, the partial pressure ratio of the two monomers fed in the reactor was controlled by the flow rate of carrier gas (i.e. Ar, Air liquide, France), which was regulated and measured by electronic mass flow controllers (MKS instruments). The partial pressure in the reactor of $\varepsilon-C L$ and DEGME exhibited linear correlations with the flow rate of argon gas and were comparable with each other [12]. Plasma co-polymerization of organic monomers was carried out on CDHA and $\beta$-TCP discs for the preliminary study on the layer characteristics, as well as on SVA-loaded macroporous CDHA and $\beta$-TCP scaffolds. For the current study, the total flow rate was varied from 20 to 25 standard cubic centimeter per minute (SCCM) by keeping the operating pressure constant at 0.5 mbar. Two operating modes were investigated to deposit the plasma coatings: Continuous Wave (CW) or Pulse Wave (PW).

Copolymer coatings were deposited at $20 \mathrm{~W} \mathrm{CW}$ plasma for $20 \mathrm{~min}$ or $90 \mathrm{~min}$ followed by deposition of a copolymer in a $1 \mathrm{~W}$ PW plasma for 5 min to improve the surface wettability. The pulsed plasma discharge coatings were performed for 20 min, the peak power $\left(\mathrm{P}_{\mathrm{pk}}\right)$ was $25 \mathrm{~W}$ and the duty cycle $\left(\mathrm{DC}=\left(\mathrm{t}_{\mathrm{on}} /\left(\mathrm{t}_{\mathrm{on}}+\mathrm{t}_{\mathrm{off}}\right)\right.\right.$, where $\mathrm{t}_{\mathrm{on}}$ and $\mathrm{t}_{\mathrm{off}}$ were the 'plasma ON' and 'plasma OFF' times respectively) was $4 \%$ to obtain the effective

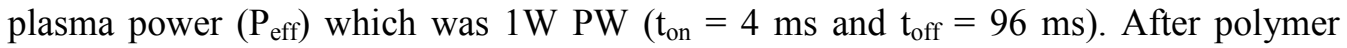
deposition, the reactor was again evacuated to base pressure before the plasma polymerization system was vented to atmospheric pressure with air. 


\subsubsection{Characterization of Plasma Polymerized Coatings}

\section{X-ray Photoelectron Spectroscopy}

The chemical composition was analyzed by XPS with a SPECS (Germany) using an Al non monocromatic source XR50 (200W and $14 \mathrm{kV}$ ) with an analyzer Phoibos 150 MCD-9 with pass energy of $25 \mathrm{eV}$, high resolution steps of $0.1 \mathrm{eV}$, chamber pressure of 5.10 ${ }^{9}$ mbar and using a Flood gun FG15/40. Peak deconvolution was performed with CasaXPS software.

\section{Surface Wettability Measurements}

Sessile drop contact angle values were measured using a video capture apparatus (Digidrop GBX-3S system, France). For each measurement, $6 \mu \mathrm{L}$ of DI water droplets were dispensed onto the coated surfaces. Four measurements were carried out on each coating and resulting values were averaged.

\section{Scanning Electron Microscopy}

Surface topography of CDHA and $\beta$-TCP discs was studied by Field-Emission Scanning Electron Microscopy using a Jeol JSM-5000/5610 SEM. Samples were Pd-Ptcoated before SEM observation. Observations were carried out at $10 \mathrm{kV}$ working voltage.

\section{Focus Ion Beam}

To ascertain the thickness of the plasma polymer layer deposited on the CDHA and $\beta$ TCP surface, Focus Ion Beam tomography (FIB, Zeiss Neon40) was performed. A $30 \mathrm{x}$ $30 \times 25 \mu \mathrm{m}$ parallelepiped was coated with Pt, cut in slices and FE-SEM pictures were taken.

\section{BET}


The Specific Surface Area (SSA) of the CaPs was measured by Nitrogen adsorption according to the Brunauer-Emmet-Teller (BET) method, (BET) in an ASAP 2020 (Micromeritics).

\section{Mercury intrusion porosimetry}

Mercury Intrusion Porosimetry (MIP, AutoPore IV, Micromeritics, USA) was performed to determine the porosity and pore entrance size distribution within the materials.

\section{Drug incorporation}

$\mathrm{CaP}$ scaffolds were loaded with SVA by immersion in $200 \mu \mathrm{g} \mathrm{SVA} / \mathrm{ml}$ solution (1 $\mathrm{mL} /$ cylinder) during $2 \mathrm{~h}$ in the case of CDHA or $24 \mathrm{~h}$ in the case of $\beta$-TCP to achieve equilibration maximum impregnation. The $\mathrm{CaP}$ scaffolds were subsequently freezedried.

\section{Drug release experiments}

CDHA and $\beta$-TCP scaffolds loaded with SVA and plasma-coated as described were immersed in $3 \mathrm{ml}$ of Phosphate Buffer Saline (PBS, $\mathrm{pH}=7.4$ ) at $37^{\circ} \mathrm{C}$ with continuous stirring. $350 \mu \mathrm{L}$ of sample was withdrawn at different time points and replaced by 350 $\mu \mathrm{L}$ of fresh PBS.

The amount of drug released was obtained by measuring the release media collected at each time point by High performance liquid chromatography (HPLC) in a Shimadzu HPLC system. A mobile phase of Acetonitrile: 0.1\% Phosphoric acid at a ratio of 50:50 (v/v), at a flow rate of $0.8 \mathrm{ml} / \mathrm{min}$ was passed through a $\mathrm{C} 8$ column (Shim-pack, 
Shimadzu). An injection volume of $10 \mu$ of the release sample was measured by photodiode array (PDA) at $\lambda_{\max }=238 \mathrm{~nm}$.

\section{Results}

\section{Characterization of the plasma polymer layer}

In this work two $\mathrm{CaP}$ materials were employed: $\mathrm{CDHA}$ obtained by low-temperature setting of a calcium phosphate cement, and $\beta$-TCP ceramic, obtained by sintering the former at high temperature. Both kinds of materials were subjected to low temperature plasma polymerization of a biocompatible and biodegradable copolymer mixture of PCL-co-PEG 1:4. As shown in Fig. 1 a) and b) the microstructure of the two substrates was significantly different, just as the SSA, which was much higher for CDHA $\left(\mathrm{SSA}_{\mathrm{CDHA}}=20 \mathrm{~m}^{2} / \mathrm{g}\right)$ than for $\beta-\mathrm{TCP}\left(\mathrm{SSA}_{\beta-\mathrm{TCP}}=0.60 \mathrm{~m}^{2} / \mathrm{g}\right)$. The scanning electron micrographs obtained on the surface of the materials (Fig. 1) revealed the typical microporous structure of $\beta$-TCP with its sintering grains, while CDHA consisted of entangled platelet-like crystals derived from the dissolution-precipitation responsible for cement hardening.

In the first place it was of interest to characterize the plasma polymer layer deposited with $\mathrm{CW}$ discharge on the two materials at the longest time evaluated of $90 \mathrm{~min}$.

FTIR measurements were performed on material obtained from the top surface of the CaPs. All materials tested display phosphate vibrations (Supplementary material 1), where the wide, strong bands correspond to $v_{3}$ stretching $\left(944-1122 \mathrm{cpom}^{-1}\right)$ and $v_{4}$ bending $\left(545-640 \mathrm{~cm}^{-1}\right)$ typical of calcium phosphates or apatites $[18,19]$. On the plasma coated materials, $\beta$-TCP-90CW and CDHA-90CW, different bands indicated the presence of the coating, which were more evident in $\beta$-TCP-90CW. In particular, bands 
were recorded at $1716 \mathrm{~cm}^{-1}$, which were attributed to the $\mathrm{C}=\mathrm{O}$ stretching vibrations of the ester carbonyl group from PCL; C-H stretching bonds were centered at 2950 and $2870 \mathrm{~cm}^{-1}$. The $\mathrm{C}-\mathrm{H}$ bending was observed at 1380 and $1460 \mathrm{~cm}^{-1}$. The weak absorption band at $3430 \mathrm{~cm}^{-1}$ was assigned to terminal $-\mathrm{OH}$ groups in the coatings which indicated the presence of polar groups incorporated into the copolymer structures [12], as -OH groups from hydroxyapatite usually appear at higher bending energies $3567 \mathrm{~cm}^{-1}$ [20].

Modifications on the surface energy and surface chemistry were recorded by means of static contact angle and XPS measurements, respectively (Table 1). Both untreated materials were very hydrophilic and due to their inherent porosity absorbed water very fast, not allowing contact angle measurement. However, after plasma polymerization water was no longer absorbed and the contact angle rose to hydrophobic values above $110^{\circ}$ in both materials, showing the highest values for $\beta-\mathrm{TCP}-90 \mathrm{CW}$.

The surface chemistry of the untreated CaPs as measured by XPS correlated adequately with their nominal composition. The polymerization treatment with PCL-co-PEG led to a decrease in the $\mathrm{O} / \mathrm{C}$ ratio, showing the presence of polymer on the surface. The layer deposited in the case of $\beta$-TCP-90CW was thicker than the detection limit of the XPS $(10 \mathrm{~nm})$, as $\mathrm{Ca}$ and $\mathrm{P}$ species were no longer detected by the technique. In the case of CDHA-90CW both $\mathrm{Ca}$ and $\mathrm{P}$ were detected but in very small quantities which could indicate that the CDHA is not completely covered by the plasma polymer. However, such low values measured can be also ascribed to background noise, so these particular $\mathrm{Ca}$ and $\mathrm{P}$ values presented in Table 1 should be taken with precaution.

Decomposition of $\mathrm{C} 1 \mathrm{~s}$ core level spectra of the plasma polymerized samples (Table 2, Figure 2) showed a profile clearly including 3 peaks corresponding to: $\mathrm{C}-\mathrm{C}$ and/or $\mathrm{C}-\mathrm{H}$ 
$285.00 \mathrm{eV}$, which were the most abundant groups, followed by $\mathrm{C}-\mathrm{O}$ at $286.63 \mathrm{eV}$ (in ether groups) and $\mathrm{C}=\mathrm{O} 288.63 \mathrm{eV}$ (probably carboxylic/ester groups). The presence of these bonds reflected the presence of the copolymer PCL: PEG 1:4. The contribution of the $\mathrm{C}=\mathrm{O}$ peaks was slightly lower in $\beta$-TCP than in CDHA, in both cases up to around $6 \%$.

As displayed in the SEM images (Figure 1), after plasma polymerization with PCL-coPEG the structure of the CaPs was covered by a layer following the original patterns of the materials, forming bush-like structures on $\beta$-TCP-90CW and worm-like structures for CDHA-90CW.

Transversal sections obtained by FIB revealed that, as anticipated by XPS measurements, the plasma polymer deposited on $\beta$-TCP-90CW was thicker (1496 \pm $162 \mathrm{~nm})$ than that deposited on CDHA-90CW $(628 \pm 61 \mathrm{~nm})$, in the same experimental conditions.

Once demonstrated that it was possible to produce PCL-co-PEG coatings on the surface of flat CDHA and $\beta$-TCP discs, the possibility to obtain polymer layers on the surface of 3D macroporous samples, herein designated as $\mathrm{CaP}$ scaffolds, was assessed. As shown in the transversal section of a CaP scaffold (Figure 3 left), both $\beta$-TCP and CDHA scaffolds contained macropores of $80 \mu \mathrm{m}$ of pore entrance size (Figure 4), with a smaller volume of pores around $2 \mu \mathrm{m}$ corresponding to the distance between sintering necks / crystal aggregates. The plateau at the nanoscale range is only present in CDHA scaffolds and corresponds to intercrystal distance. The total porosity was of $81.5 \%$ for $\beta$-TCP and of $82.2 \%$ for CDHA. Plasma polymerization for $90 \mathrm{CW}$ min showed the same bush-like and worm-like structures in the pores located at the top surface of the samples (Figure 3, right). In addition, it was observed that for $\beta$-TCP, the plasma 
process evaluated here was capable of depositing PCL-co-PEG coatings up to a depth of $550 \mathrm{~mm}$ by diffusion through the interconnected macropores of the material. This was not visible for CDHA samples, where the coating was restricted to the outer surface.

Drug loading and release

$\mathrm{CaP}$ Scaffolds were drug-loaded by immersion in a solution containing SVA. SVA showed high affinity for CDHA as high loadings of $168 \mu \mathrm{g} / \mathrm{scaffold}$ were obtained in only two hours (Table 3 ), which accounts for $84 \pm 6 \%$ loading efficiency. As $\beta$-TCP scaffolds displayed lower affinity they were allowed $24 \mathrm{~h}$ to equilibrate in the SVA solutions. Anyhow, the loading efficiency was much lower $(9 \pm 2 \%)$ so the quantity of drug loaded in $\beta$-TCP was of $18.78 \mu \mathrm{g}$.

The drug release pattern for the SVA-loaded $\beta$-TCP and CDHA either untreated or after 90 min of CW plasma coating is shown in Figure 5. Despite these loading diferences, the amount of drug released was very similar between the untreated $\beta$-TCP and CDHA scaffolds. Over nearly 2 weeks ( $284 \mathrm{~h}$ ) both untreated materials showed a progressive release, while the coated samples dislayed different behavior: $\beta$-TCP-SVA-90CW did not show any drug release along 2 weeks of experiment, while in the case of CDHA release was initially blocked, delayed for the first $2 \mathrm{~h}$, and release progressed slowly afterwards.

Once it was established that the plasma coatings were effective in blocking drug release, shorter treatment times and pulsed mode depositions were evaluated. All conditions evaluated completely blocked SVA release from $\beta$-TCP scaffolds. On the contrary, CDHA scaffolds coated with shorter polymerization times of 20 min in continuous wave mode (Fig. 6 a) displayed controlled release properties over the 
course of the experiment. Plasma polymers produced in pulsed wave mode showed to be more efficiently blocking the surface in 20 min than $\mathrm{CW}$ polymers (Fig. 6 b), as release was delayed for $5 \mathrm{~h}$ and progressed slowly afterwards.

\section{Discussion}

To overcome the intrinsic limitations of $\mathrm{CaPs}$ to regulate drug release, this paper focuses on the use of biocompatible plasma-polymers to provide a tool to control drug release from drug-loaded $\mathrm{CaP}$ scaffolds with very complex surfaces and intricate 3D structures. One advantage of using a dry method such as plasma lies in avoiding, for instance, any ion dissolution of the calcium phosphates and thus conserving intact their initial properties. If the material has already been loaded with drugs, coating them is a complicated issue, as any contact with processing solutions would lead to dissolution/diffusion of the drugs and thus loss active principle from the biomaterial.

In this work, it has been shown that plasma polymers (PCL-co-PEG) can be deposited on the surface of $\mathrm{CaP}$ cements and ceramics with very different surface features. As shown by the IR spectra (Figure 1), the bands corresponding to $\mathrm{C}-\mathrm{H}, \mathrm{C}-\mathrm{O}$ and $\mathrm{OH}$ confirm the formation of a polymer layer on the surface of the ceramics. The thickness of this polymer layer is variable depending on the material used, i.e. $\beta$-TCP ceramics or CDHA cements which have different morphologies and specific surface area (SSA). This was confirmed both by XPS (Table 1), in which the Ca and P species were not detectable - or hardly - after the CW plasma polymerization treatment for $90 \mathrm{~min}$, and also by SEM-FIB (Figure 2), which allowed measuring the thickness of the layers. Thus, $\beta$-TCP showed a layer which was two times thicker than the one deposited on CDHA in the same conditions. This can be attributed to the great differences in SSA between both materials (CDHA has 33 times higher SSA than $\beta$-TCP), which is related 
to their structure, consisting in CDHA platelet-like crystals in one case, and flat porous structures typical of sintered materials in $\beta$-TCP. The thickness of the layer obtained by CW plasma discharge for 90 min was much larger than that recorded in previous works [12], mainly due to the longer treatment times employed. The thickness of the coating (1.49 \pm 0.16 and $0.63 \pm 0.06 \mu \mathrm{m}$ on $\beta$-TCP and CDHA respectively) led to important changes in the surface topography of the CaP materials, leading to the formation of bush or worm-like structures. This change in surface morphology, together with the change in the chemical nature of the surface is expected to result in significant changes in the in vitro and in vivo biological performance of the material [9], which will have to be further investigated.

The chemical structure of $\beta$-TCP and CDHA is $\mathrm{Ca}_{3}\left(\mathrm{PO}_{4}\right)_{2}$, and $\mathrm{Ca}_{9}\left(\mathrm{HPO}_{4}\right)\left(\mathrm{PO}_{4}\right)_{5}(\mathrm{OH})_{2}$ respectively, with a theoretical $\mathrm{Ca} / \mathrm{P}$ ratio of 1.5 for both compounds [21]. None of the materials itself contains carbon, so its presence on the surface of the samples (Table 1) indicates an alteration of the surface chemistry, which could be ascribed to contamination by adsorbed hydrocarbons. The PCL-co- PEG 1:4 plasma coatings (90 $\mathrm{CW}$ ) deposited on the surface had a $0.3 \mathrm{O} / \mathrm{C}$ ratio in both $\beta$-TCP and CDHA, which was close to that of Bhatt et al. [9] $(0.36 \mathrm{O} / \mathrm{C})$, who employed different deposition conditions (mainly treatment time and substrates employed) (Table 1). Moreover, the coatings led to a significant change in the wetting properties of the materials; both CaPs were initially hydrophilic, with fast water absorption through the pores of the materials. After plasma polymerization these relatively low $\mathrm{O} / \mathrm{C}$ ratios, together with blocking of the surface porosity, raised the surface hydrophobicity of the materials, which displayed contact angles between $115^{\circ}$ and $121^{\circ}$. The high roughness of the materials, generated 
in the growth of the plasma polymer on the intricate crystalline structures of the $\mathrm{CaPs}$ must also be related to these considerable differences in the wettability measurements.

In the second part of the work, 3D CaP scaffolds made of $\beta$-TCP or CDHA were studied. These are the macroporous counterparts of the $\beta$-TCP and CDHA evaluated in the first part of the work, thus preserving their microstructure (Figure $1 \mathrm{a}$ and $\mathrm{b}$ ) but with additional interconnected macroporosity (Figure 3 left). The advantage of introducing macroporosity to $\mathrm{CaPs}$ is envisaged as a method to facilitate bone ingrowth not only from the external surface but throughout the bulk of the material [22]. This would accelerate its resorption and transformation into newly formed bone tissue [23]. It has also been shown that macroporosity is relevant for drug delivery, as it allows improving fluid flow throughout the material [5].

In the $3 \mathrm{D}$ treatment of the macroporous $\mathrm{CaP}$ scaffolds it was shown here that the plasma polymer coating can be performed successfully on the materials, and that the interconnections between the macropores allow the penetration of the plasma polymer inside the scaffold (Figure 3). This is particularly evident in the case of $\beta$-TCP, where the characteristic bush-like structures can be observed both on the surface, and inside pores of the scaffold down to a depth of $550 \mu \mathrm{m}$ (Figure 3). The higher SSA of CDHA was probably related to the fact that the coating layer was restricted to the top surface of the CaP scaffolds, and no penetration was observed by SEM, as possibly all available monomer was already consumed in the coating of the wider surface.

In the subsequent steps, the drug release of the plasma-coated scaffolds was evaluated. Simvastatin acid (SVA) was incorporated to the CaP scaffolds. Simvastatin is a commonly used cholesterol-lowering drug which has been shown to stimulate osteogenesis locally through an up-regulation of BMP-2 expression [24] and it has also 
been related to dose-dependent improved angiogenesis [25]. Enhancing blood vessel growth within the scaffolds is of interest with views on promoting scaffold resorption and bone regeneration.

The adsorption of SVA, which is a hydrophilic drug (Eq. 1), was much favoured in CDHA scaffolds than in $\beta$-TCP (table 3). This is reflected in drug loading of $\beta$-TCP being only $11 \%$ of that of CDHA - even after $\beta$-TCP samples allowed to equilibrate with the loading solution for $24 \mathrm{~h}$ in comparison to $2 \mathrm{~h}$ for CDHA scaffolds. As both kinds of scaffolds have very close porosity around $82 \%$, this difference can probably be attributed to the higher SSA of CDHA but also to its different chemistry, which can probably allow for the creation of hydrogen bonds with SVA.

Drug release patterns were significantly modified by the PCL-co-PEG plasma coatings deposited (Figs. 5 and 6). On the one hand, uncoated $\beta$-TCP scaffolds showed a continuous progressive release for the first $5 \mathrm{~h}$ where $98 \%$ of the initial drug loads was released. After 3 days the drug in the media started degrading (shown by a decrease of the measured drug in the media) (Fig. 5a). None of the $\beta$-TCP scaffolds plasma coated with the PCL-co-PEG in different conditions showed any SVA release in the evaluated timeframe of 11 days (Data not shown - Scheme shown in Fig. 5a). This is indicative of the lack of diffusion of SVA through the thick plasma layer deposited on the $\beta$-TCP, shown to be much thicker than that of CDHA in the $90 \mathrm{CW}$ coated samples. It is important to recall that the amount of SVA loaded into $\beta$-TCP is an order of magnitude lower than that of CDHA, so even thinner coatings of 20CW (Supplementary material 2) are able to block release from $\beta$-TCP.

In contrast, untreated CDHA scaffolds showed continuous release for more than 10 days, although the amount released was only around $16 \%$ of the initial SVA loading 
(Fig. 5b). The 90 CW PCL-co-PEG layer on the drug-loaded CDHA scaffolds was three times thinner than that of $\beta$-TCP. Initially (first $1.5 \mathrm{~h}$ ) it blocked SVA release and subsequently allowed its slow diffusion along the timeframe of the experiment. Shorter treatment times of 20 min (CDHA-SVA-20CW) presented the same profile (Fig. 6a), and though release started from the beginning of the experiment, the rate of release was slowed down with respect to the untreated CDHA scaffolds. This is in agreement with a previous work, where Bhatt et al. [12] showed that by gradually increasing the barrier layer thickness with longer plasma deposition times, the amount of a dye released was reduced.

In parallel, the mode of deposition clearly affected the drug release, as only $20 \mathrm{~min}$ of treatment in pulsed mode (CDHA-SVA-20PW) were able to block the release for the initial $5 \mathrm{~h}$, allowing afterwards for sustained release (Fig. 6b). This is most probably related to a lower extent of monomer fragmentation achieved by pulsed discharges, with higher retention of ether functional groups $[26,27]$. Plasma phase fragmentation plays an important role in determining the deposition rate and surface chemistry of the deposited film and depends on the kind of monomer [28].

The drug delivery matrices developed in this work constitute complex structures, which combine: i) a highly porous (macro, micro and nanoporous) non-degrading bulk [3] which was shown to release drugs (ie. antibiotics) through complex mechanisms following non-Fickian diffusion [5] with ii) a biodegradable plasma polymer coating. The mechanisms involved in drug release from this kind of coatings on flat surfaces were shown to range from zero-order to non-Fickian diffusion as the coating thickness increased [12]. Thus, the drug release in the developed dosage forms is expected to be 
ruled by the coating until it is completely degraded, followed by diffusion from the $\mathrm{CaP}$ matrix afterwards.

\section{Conclusions}

This work has allowed demonstrating that it is possible to fine-tune drug release from 3D ceramic scaffolds (CDHA \& $\beta$-TCP) with different and very complex surface textures by a dry plasma polymerization process by employing biocompatible plasma polymers, depending on the treatment conditions. The thickness and structure of the PCL-co-PEG (1:4) polymer layer obtained is highly dependent on the texture of the materials, in particular on its SSA. Thicknesses of up to $1.4 \mu \mathrm{m}$ were obtained on $\beta$ $\mathrm{TCP}$, and the plasma polymers were able to penetrate the macroporous structure of $3 \mathrm{D}$ calcium phosphate foams, up to a certain depth. The topography and chemistry of the materials were significantly modified by the coatings which led to nano-rough structures which could explain the low wettability measured on the materials.

Simvastatin acid (SVA) was incorporated to the calcium phosphate scaffolds of $\beta$-TCP and CDHA as osteogenic and angiogenic drug, and the plasma coatings were useful to modulate the drug release: thicker PCL-co-PEG layers acted as barriers on $\beta$-TCP foams impairing SVA release throughout the whole release experiment (11 days), while on CDHA the presence of thinner polymer layers - due to its much higher SSA allowed for the diffusion of the drug, leading to progressive and controlled drug release with respect to the uncoated biomaterial. On CDHA, longer treatment times (90 min in continuous wave plasma discharges) implied initially blocking of release followed by a slow release pattern, while shorter times $(20 \mathrm{~min})$ allowed a slow, controlled release. Pulsed wave plasma discharges allowed efficiently depositing plasma polymer layers 
which could initially block the release, in shorter times $(20 \mathrm{~min})$. Thus, the plasma coatings evaluated have shown to be useful tools for the tuning of drug release from bone biomaterials, allowing to produce ceramic dosage forms combining delayed release, followed by controlled delivery of drugs.

\section{ACKNOWLEDGEMENTS}

Authors acknowledge the Spanish Government for financial support through Project MAT2012-38438-C03-01, co-funded by the EU through European Regional Development Funds, and Ramon y Cajal fellowship of CC. Support for the research of MPG was received through the "ICREA Academia" prize for excellence in research, funded by the Generalitat de Catalunya. The European Commission is acknowledged for support through Cost Action MP1101 and the University Pierre et Marie Curie for the $\mathrm{PhD}$ scholarship.

\section{REFERENCES}

[1] Ginebra M. P., Espanol M., Montufar E. B., Perez R. A., Mestres G., New processing approaches in calcium phosphate cements and their applications in regenerative medicine, Acta Biomater. 6 (8) (2010) 2863-2873.

[2] LeGeros R. Z., Calcium phosphate-based osteoinductive materials, Chem. Rev., 108 (11) (2008) 4742-4753.

[3] Ginebra M.P., Canal C., Espanol M., Pastorino D., Montufar E. B., Calcium phosphate cements as drug delivery materials, Adv. Drug Deliv. Rev., 64(12) (2012) 1090-1110.

[4] Canal C., Pastorino D., Mestres G., Schuler P., Ginebra M.P. Relevance of microstructure for the early antibiotic release of fresh and pre-set calcium phosphate cements. Acta Biomater. 9 (2013) 8403-12. 
[5] Pastorino D., Canal C., Ginebra M.P., Drug delivery from injectable calcium phosphate foams by tailoring the macroporosity-drug interaction, Acta Biomater., 12 (2015) 250-259.

[6] Maazouz Y., Montufar E. B., Guillem-Marti J., Fleps I., Ohman C., Persson C., Ginebra M. P., Robocasting of biomimetic hydroxyapatite scaffolds using self-setting inks, J. Mater. Chem. B, 2 (2014) 5378-5386.

[7] Labay C., Canal C., García-Celma M. J., Influence of Corona Plasma Treatment on Polypropylene and Polyamide 6.6 on the Release of a Model Drug, Plasma Chem. Plasma Process., 30 (6) (2010) 885-896.

[8] Labay C., Canal J. M., Canal C., Relevance of Surface Modification of Polyamide 6.6 Fibers by Air Plasma Treatment on the Release of Caffeine, Plasma Process. Polym., 9(2) (2012) 165-173.

[9] Szili E.J., Short R.D., Steele D.A., Bradley J.W., Surface modification of biomaterials by Plasma Polymerization, in: Surface Modification of Biomaterials; Methods, Analysis and Applications, First ed. Woodhead Publishing Limited, 2011, pp. 3-39.

[10] Simovic S., Losic D., Vasilev K., Controlled drug release from porous materials by plasma polymer deposition, Chem. Commun., 46(8) (2010) 1317-1319.

[11] Vasilev K., Poulter N., Martinek P., Griesser H.J., Controlled release of levofloxacin sandwiched between two plasma polymerized layers on a solid carrier, ACS Appl. Mater. Interfaces, 3 (12) (2011) 4831-4836.

[12] Bhatt S., Pulpytel J., Mirshahi M., Arefi-Khonsari F., Plasma co-polymerized nano coatings - As a biodegradable solid carrier for tunable drug delivery applications, Polymer, 54(18) (2013) 4820-4829.

[13] Montazerolghaem M., Enqvist H., Karlson Ott M., Sustained release of simvastatin from premixed injectable calcium phosphate cement, J. Biomed. Mater. Res. A, 102 (2014) 304-307. 
[14] Yin H., Li Y.G., Si M., Li J.M., Simvastatin-loaded macroporous calcium phosphate cement: preparation, in vitro characterization, and evaluation of in vivo performance, J. Biomed. Mater. Res. A, 100 (2012) 2991-3000.

[15] Kaesemeyer W.H., Caldwell R.B., Huang J., Caldwell R.W., Pravastatin sodium activates endothelial nitric oxide synthase independent of its cholesterol-lowering actions, J. Am. Coll. Cardiol., 33(1) (1999) 234-241.

[16] Bhatt S., Pulpytel J., Mirshahi M., Arefi-Khonsari F., Catalyst-Free PlasmaAssisted Copolymerization of Poly(e-caprolactone)-poly(ethylene glycol) for Biomedical Applications, ACS Macro Lett., 1(6) (2012) 764-767.

[17] Bhatt S., Pulpytel J., Mirshahi M., Arefi-Khonsari F., Nano thick poly( $\varepsilon-$ caprolactone)-poly(ethylene glycol) coatings developed by catalyst-free plasma assisted copolymerization process for biomedical applications, RSC Adv., 2 (2012) 9114-9123.

[18] Mestres G., Le Van C., Ginebra M.P., Silicon-stabilized $\alpha$-tricalcium phosphate and its use in a calcium phosphate cement: characterization and cell response, Acta Biomater., 8 (2012) 1169-1179.

[19] Radin S. R., Ducheyne P., The effect of calcium phosphate ceramic composition and structure on in vitro behavior. II. Precipitation., J. Biomed. Mater. Res., 27 (1993) $35-45$.

[20] Antonakos A., Liarokapis E., Leventouri T., Micro-Raman and FTIR studies of synthetic and natural apatites., Biomaterials, 28 (2007) 3043-3054.

[21] Ginebra M.P., Fernandez E., De Maeyer E.A.P., Verbeeck R.M.H., Boltong M.G., Ginebra J., Driessens F.C.M., Planell J.A., Setting Reaction and Hardening of an Apatitic Calcium Phosphate Cement, J. Dent. Res., 76 (1997) 905-912.

[22] Montufar E.B., Traykova T., Gil C., Harr I., Almirall A., Aguirre A., Engel E., Planell J.A., Ginebra M.P., Foamed surfactant solution as a template for self-setting injectable hydroxyapatite scaffolds for bone regeneration, Acta Biomater., 6 (2010) 876-885. 
[23] Karageorgiou V., Kaplan D., Porosity of 3D biomaterial scaffolds and osteogenesis, Biomaterials, 26 (2005) 5474-5491.

[24] Mundy G., Stimulation of Bone Formation in Vitro and in Rodents by Statins, Science, 286(5446) (1999) 1946-1949.

[25] Weis M., Statins Have Biphasic Effects on Angiogenesis, Circulation, 105 (2002) 739-745.

[26] Buxadera-Palomero J., Canal C., Torrent-Camarero S., Garrido B., Gil F.J., Rodríguez D., Antifouling coatings for dental implants: Polyethylene glycol-like coatings on titanium by plasma polymerization, Biointerphases, 10(2) (2015) 029505.

[27] Labay C., Canal J.M., Modic M., Cvelbar U., Quiles M., Armengol M., Arbos M.A., Gil F.J., Canal C., Antibiotic-loaded polypropylene surgical meshes with suitable biological behaviour by plasma functionalization and polymerization, Biomaterials, 71 (2015) 132-144.

[28] Michelmore A., Gross-Kosche P., Al-Bataineh S.A., Whittle J.D., Short R.D., On the effect of monomer chemistry on growth mechanisms of nonfouling PEG-like plasma polymers, Langmuir, 29 (2013) 2595-2601. 
Tables

Table 1. Static contact angle and X-ray photoelectron spectroscopy (atomic concentration and atomic ratio) of the different $\mathrm{CaP}$ discs either untreated of after plasma polymerization

\begin{tabular}{|c|c|c|c|c|c|c|c|}
\hline & \multirow{2}{*}{$\boldsymbol{\theta}_{\mathbf{s}}\left({ }^{\boldsymbol{}}\right)$} & \multicolumn{4}{|c|}{ Atomic concentration \% } & \multicolumn{2}{c|}{ Atomic ratio } \\
\cline { 3 - 8 } & $*$ & $\mathbf{C 1 s}$ & $\mathbf{O 1 s}$ & $\mathbf{C a 2 p}$ & $\mathbf{P 2 p}$ & $\mathbf{O} / \mathbf{C}$ & $\mathbf{C a} / \mathbf{P}$ \\
\hline $\boldsymbol{\beta}$-TCP & $*$ & 14.83 & 53.61 & 19.54 & 11.98 & 3.61 & 1.63 \\
\hline $\boldsymbol{\beta}$-TCP-90CW & $121 \pm 2$ & 76.99 & 23.01 & -- & -- & 0.29 & -- \\
\hline CDHA & $*$ & 10.51 & 57.81 & 19.05 & 12.62 & 5.50 & 1.51 \\
\hline CDHA-90CW & $115 \pm 1$ & 76.09 & 23.75 & $0.12 * *$ & $0.04 * *$ & 0.30 & $--* *$ \\
\hline
\end{tabular}

*Not possible to measure contact angle due to too fast water absorption.

** Too small quantities to be taken with precaution. 
Table 2. Cls peak deconvolution

\begin{tabular}{|l|c|c|c|}
\hline & \multicolumn{3}{|c|}{ Atomic percentage } \\
\hline & \multicolumn{3}{|c|}{ C1s } \\
\hline & $\mathbf{2 8 5 . 0 0} \boldsymbol{e V}$ & $\mathbf{2 8 6 . 6 3} \boldsymbol{e V}$ & $\mathbf{2 8 8 . 6 4} \boldsymbol{e V}$ \\
$\mathrm{C}-\boldsymbol{C}, \boldsymbol{C}-\boldsymbol{H}$ & $\boldsymbol{C}-\boldsymbol{O}$ & $\boldsymbol{C}=\boldsymbol{O}$ \\
\hline$\beta$-TCP-90CW & 55.7 & 37.9 & 6.4 \\
\hline CDHA-90CW & 51.5 & 42.4 & 6.1 \\
\hline
\end{tabular}


Table 3. Simvastatin acid loaded and final amount released (11 days) from CDHA and $\beta$-TCP scaffolds with different plasma polymerization times.

\begin{tabular}{|l|c|c|}
\hline Samples & $\mathbf{Q}_{\text {loaded }}(\boldsymbol{\mu g})$ & $\mathbf{Q}_{\text {released }}(\boldsymbol{\mu g})$ \\
\hline $\boldsymbol{\beta}$-TCP-SVA & $18.8 \pm 3.7$ & $18.4 \pm 0.4$ \\
\hline $\boldsymbol{\beta}$-TCP-SVA-20CW & $18.8 \pm 3.7$ & $0.0 \pm 0.0$ \\
\hline $\boldsymbol{\beta}$-TCP-SVA-90CW & $18.8 \pm 3.7$ & $0.1 \pm 0.1$ \\
\hline $\boldsymbol{\beta}$-TCP-SVA-20PW & $18.8 \pm 3.7$ & $1.3 \pm 0.1$ \\
\hline CDHA-SVA & $168.1 \pm 12.9$ & $27.6 \pm 11.7$ \\
\hline CDHA-SVA-20CW & $168.1 \pm 12.9$ & $2.5 \pm 2.5$ \\
\hline CDHA-SVA-90CW & $168.1 \pm 12.9$ & $4.7 \pm 0.7$ \\
\hline CDHA-SVA-20PW & $168.1 \pm 12.9$ & $2.6 \pm 2.6$ \\
\hline
\end{tabular}




\section{Figure captions}

Figure 1. SEM images of the different materials before (top images) and after plasma polymerization with PCL-PEG (4:1). $\beta$-TCP (a), $\beta$-TCP-90CW (b), CDHA (c) and CDHA-90CW (d) and FIB-SEM cross-section of the surface of both polymerized materials (e) $\beta$-TCP-90CW and (f) CDHA-90CW.

Figure 2. X-ray Photoelectron Spectroscopy C1s peak deconvolution, of (a) $\beta$-TCP and (b) CDHA with PCL-co-PEG (1:4) plasma coating 90CW.

Figure 3. Scanning electron micrographs of transversal sections of $\beta$-TCP and CDHA scaffolds after 90 min CW plasma polymerization at different depths. Views of the surface of pores found on the top surface layer (top image), at a depth of $550 \mu \mathrm{m}$, and at $1500 \mu \mathrm{m}$ (lower image).

Figure 4. Pore entrance size distribution of $\beta$-TCP and CDHA obtained by Mercury Intrusion Porosimetry.

Figure 5. Cumulative release of SVA in (\%) from $\beta$-TCP (a) or CDHA (b) scaffolds either untreated of after 90 min coating in continuous wave mode with hypothesis on the possible phenomena taking place (below).

Figure 6. Release pattern for SVA from CDHA scaffolds at (a) different treatment times with continuous wave mode $(\mathrm{CW})$ or $(b)$ different power supply mode being either continous $(C W)$ or pulsed $(P W)$. 


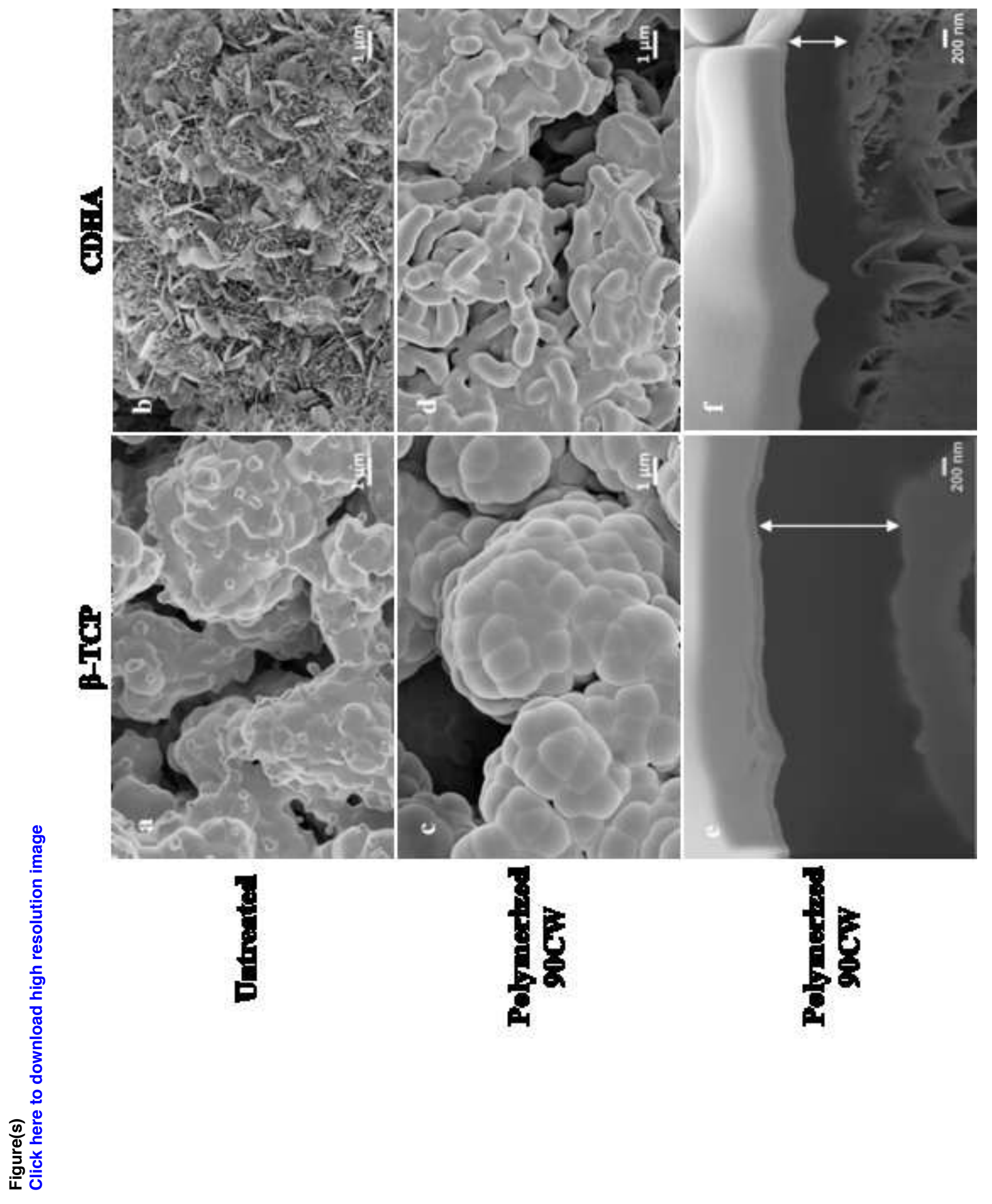




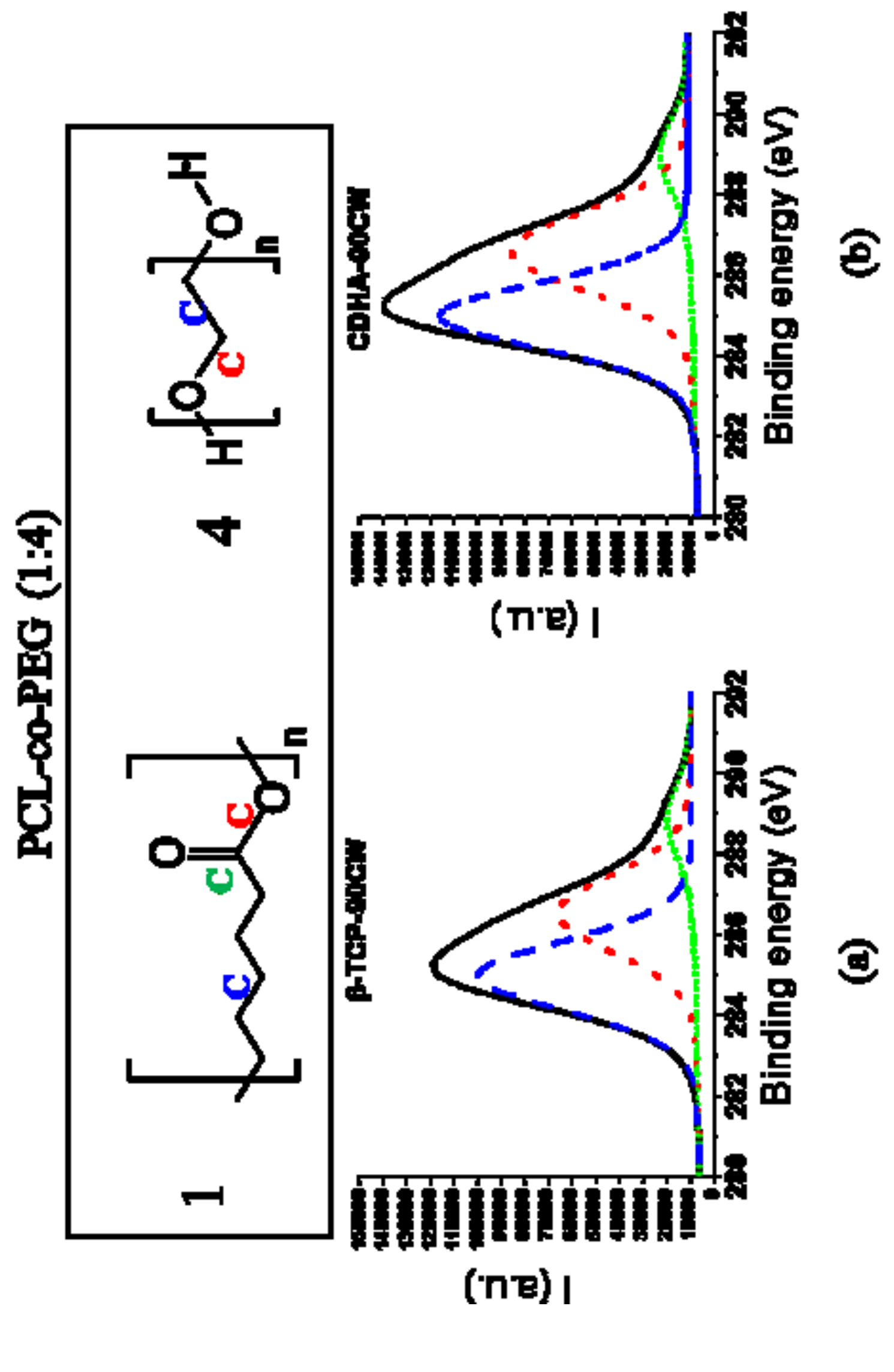



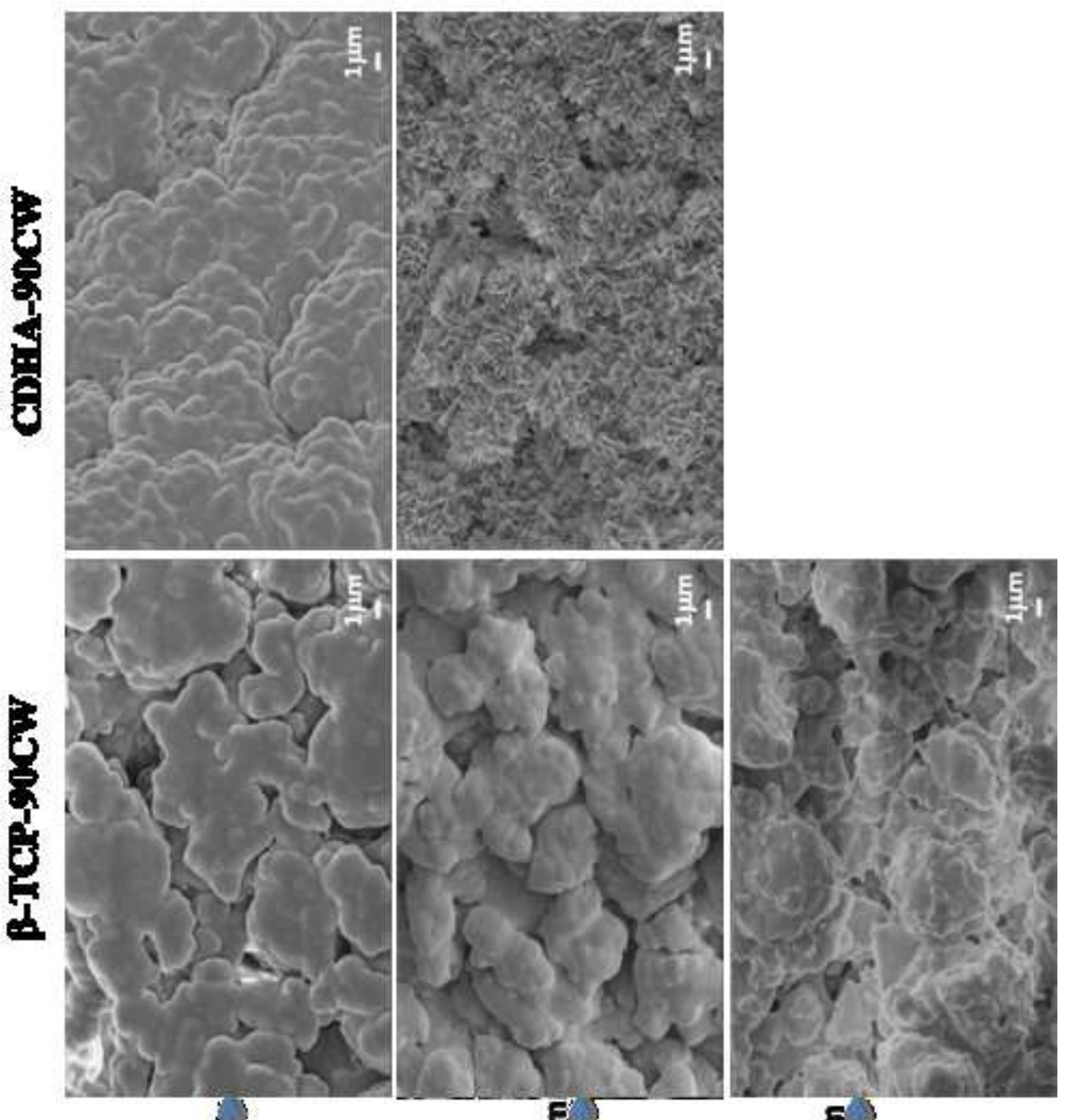









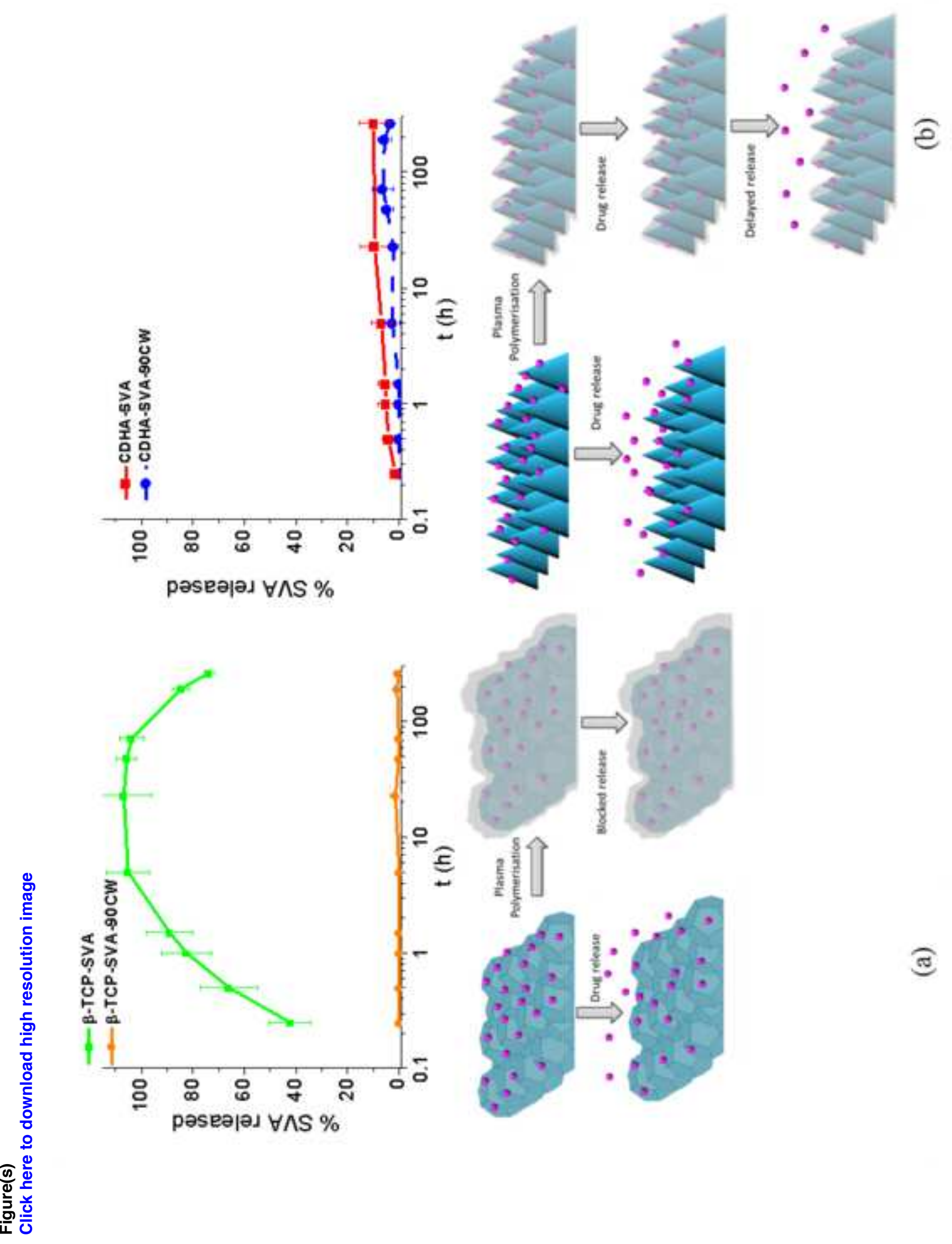


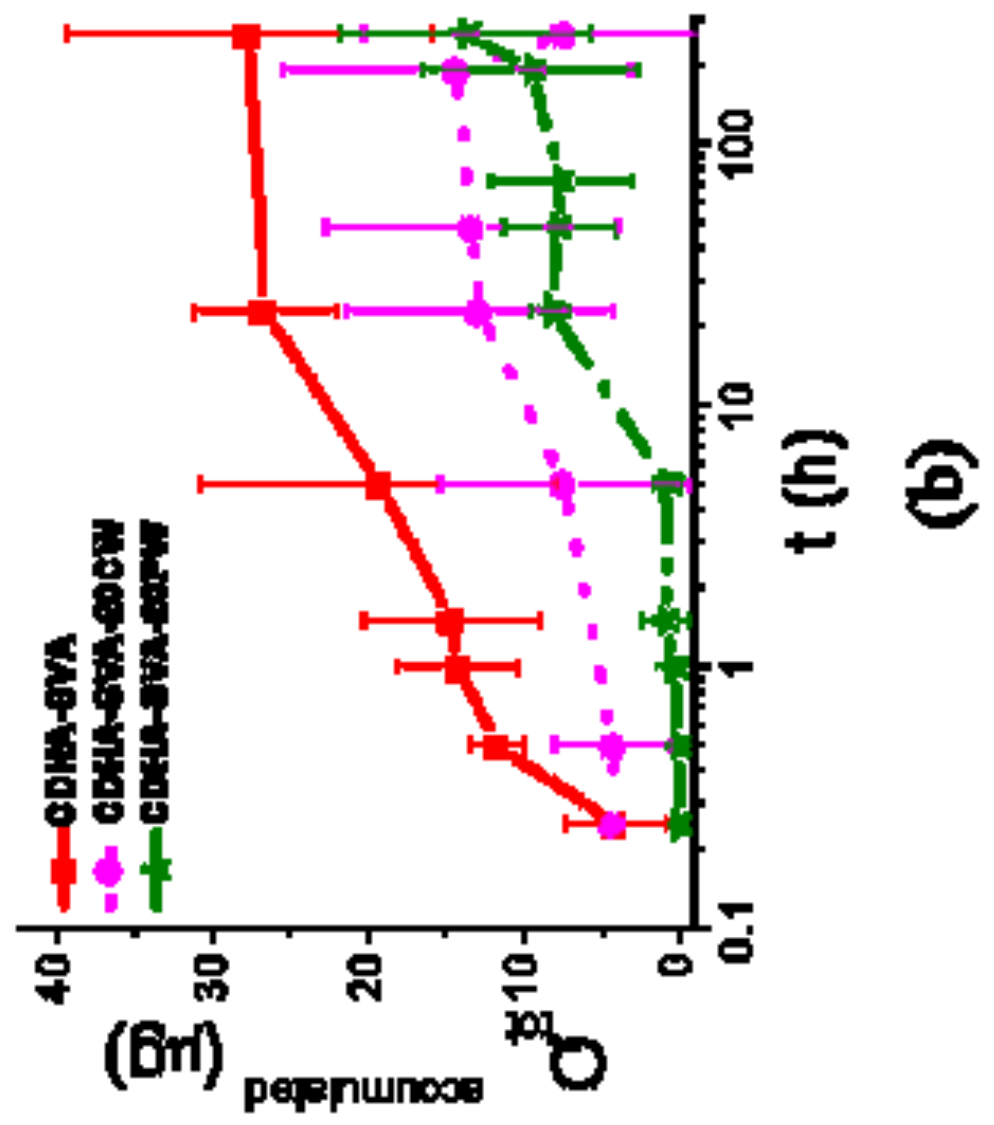

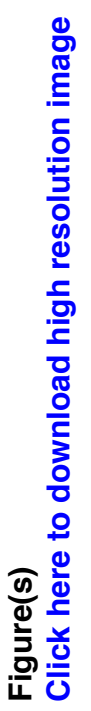

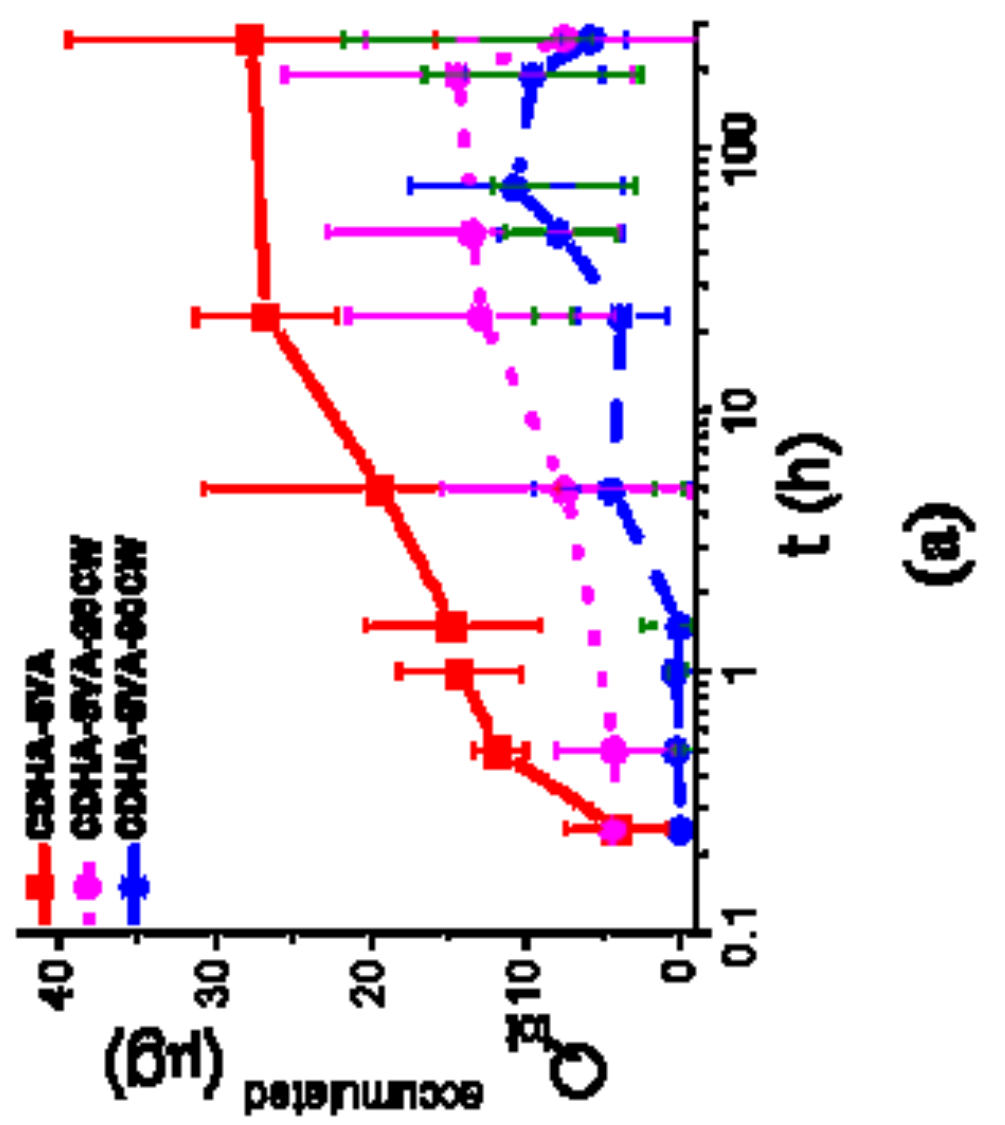

